\title{
Judging qualification, gender, and age of the observer based on gaze patterns when looking at faces ${ }^{\star}$
}

\author{
Pawel Kasprowski ${ }^{1[0000-0002-2090-335 X]}$, Katarzyna \\ Harezlak ${ }^{1[0000-0003-3573-9772]}$, Piotr Fudalej2 [0000-0001-8777-7434], and Pawel \\ Fudalej $^{3}$ \\ 1 Silesian University of Technology, Akademicka 16, Gliwice, Poland \\ pawel.kasprowski@polsl.pl \\ 2 Department of Orthodontics and Dentofacial Orthopedics, School of Dental \\ Medicine, University of Bern, Bern, Switzerland \\ 3 Private Practice, Warsaw, Poland
}

\begin{abstract}
The research aimed to compare eye movement patterns of people looking at faces with different but subtle teeth imperfections. Both non-specialists and dental experts took part in the experiment. The research outcome includes the analysis of eye movement patterns depending on the specialization, gender, age, face gender, and level of teeth deformation. The study was performed using a novel, not widely explored features of eye movements, derived from recurrence plots and Gaze Self Similarity Plots. It occurred that most features are significantly different for laypeople and specialists. Significant differences were also found for gender and age among the observers. There were no differences found when comparing the gender of the face being observed and levels of imperfection. Interestingly, it was possible to define which features are sensitive to gender and which to qualification.
\end{abstract}

Keywords: Eye tracking - Gaze patterns $\cdot$ Faces observation · Age estimation.

\section{INTRODUCTION}

By registering eye movements of people observing images, it is possible to obtain much interesting information. On the one hand, it is possible to learn something about the observers - their knowledge, intentions, anxiety, etc. On the other side, by registering eye movements of several observers looking at the same image, it is also possible to learn something about the picture - if the content is interesting, shocking, which parts of the image are the most interesting, etc.

\footnotetext{
* This work was supported by Silesian University of Technology, Rector's Pro-Quality Grant Number: 02/100/RGJ20/0002 and Statutory Research funds of Department of Applied Informatics, Silesian University of Technology, Gliwice, Poland (02/100/BK_20/0003)
} 


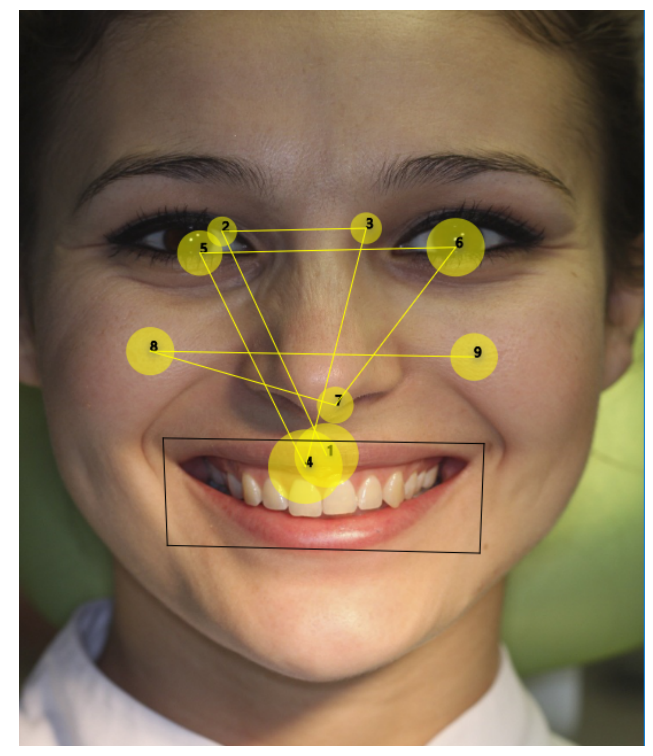

Fig. 1. A scan-path of a typical face observation

Registering eye movements while observing other people's faces is one of the most popular experiments because face observing patterns are very specific for humans. The ability to follow and analyze somebody's face is one of the first skills achieved by a newborn baby. During our whole life, we train observation of faces to recognize people's intentions, moods, etc. Lack of this ability may be considered a severe disadvantage. The analysis of eye movement patterns during face observation has been used to diagnose autism [4] or Alzheimer's disease [12]. The pattern is also supposed to be idiosyncratic [3,8], different for races [2] and age [11], and changes if the observer knows the face being observed [7].

It is well known that people typically start by looking at the other people's eyes and then look at the mouth and nose area. It is called a 'face triangle'. However, subtle differences in this pattern may reveal information about the observer and the face being observed. It is the central assumption of the research described in this paper. We presented faces of people with and without various teeth imperfections to two groups of participants: dentists and non-specialists without any connection with dentistry. Contrary to the previous research that showed that people tend to look longer at teeth when imperfections are visible [9], this time, the differences were subtle and barely distinguishable even for specialists.

Our first assumption was that eye movement patterns of dentists and laypeople will be distinguishable. We also expected that eye movement patterns would depend on the level of teeth imperfections - especially for the dentists' group. At the same time, we wanted to explore if the eye movement patterns depend on the gender and age of both people being observed and observers. 
Many papers show differences in eye movement patterns between novices and specialists, e.g., radiographers [10], or chess experts [13]. Some works were also devoted to the dentists' gaze patterns - e.g., when they looked at CT images [14]. However, to the best of our knowledge, there was no published research concerning differences between non-specialists and dentists while looking at face images. In the case of gender, some evidence of different women's and men's gaze patterns has been shown in [5] - which revealed that women are better at recognizing familiar faces.

However, most papers cited above compared gaze patterns using only traditional criteria such as the number and duration of fixations and area of interest (AOI) analysis. Our paper analyses also additional, relatively new, and unexplored measures based on recurrence plots [1] and Gaze Self-Similarity Plots [6]. Therefore, this paper's contribution is two-fold: (1) it explores the previously unchecked problem of comparing dentists with laypeople when looking at faces, and (2) it introduces novel and yet unexplored techniques to perform this comparison.

\section{METHODS AND MATERIAL}

The experimental design consisted of a presentation of 48 human faces with three levels of dental imperfections (16 faces for each level). Every face was presented for 6 seconds. The participants' task was to just freely observe the pictures and try to judge the attractiveness of the person being observed.

The same presentation was shown to 53 participants, including 25 nonspecialists and 28 dental specialists. Participants' eye movements were recorded during each trial utilizing The Eye Tribe eye tracker with a $60 \mathrm{~Hz}$ sampling rate. It gave about 360 subsequent gaze points for each 6 -second recording - called an observation in the subsequent text.

The data registered during the experiment were analyzed to remove observations with improper gaze points (e.g., when a person closed eyes for some time or turned away her eyes). Observations for which there were less than 250 gaze points were removed from the dataset. Finally, 1883 observations were available for further analyses, including 929 observations of specialists and 954 observations of non-specialists. The dataset consisted of 654 observations from women and 1229 from males, and 1188 observations of female faces and 695 observations of male faces.

Every image of the face used during the experiment was processed to define two areas of interest (AOI): mouth and incisor teeth. The time to gaze at these AOIs and the overall time of staring at them were calculated for each observation. Additionally, typical eye movement signal properties such as fixation number and average fixation duration were measured.

The next step was the calculation of four measures used to quantify eye movement recordings based on the recurrence plot defined in [1]. Given a sequence of $\mathrm{N}$ fixations: 
- Recurrence represents the percentage of recurrent fixations (i.e., how often the observer refixates previously fixated image positions).

- Determinism represents repeating fixation patterns.

- Laminarity measures how often the same areas of a scene are repeatedly fixated.

- Center of recurrence mass (CORM) measures how close the recurrent fixations points are situated.

Due to space limits, we do not present details about the way these four measures are calculated, which may be found in [1].

The next group of measures included the features derived from Gaze Self Similarity Plots (GSSP) [6]. At first, the co-occurrence matrix was calculated for every GSSP, and then the contrast, homogeneity, and uniformity were calculated.

- Homogeneity gives information to what extend nearby gazes are placed in similar locations.

- Contrast reveals long jumps (saccades) from one gaze point to another.

- Uniformity measures gaze pairs repetitions. It is high when the GSSP contains similar areas, which means that the same paired values with the same arrangement repeatedly appear in the image.

All three features were calculated for $[1,1]$ offset. Please refer to $[6]$ for calculation details.

The last group of features included the coverage - the whole image was divided into $N \times N$ areas and the coverage $N$ was calculated using the Equation 1 .

$$
C O V_{N}=\frac{\sum_{i}^{N} \sum_{j}^{N}(\exists F i x \in \operatorname{area}(i, j)) ? 1: 0}{N \times N}
$$

All examined features are summarized in Table 1.

\section{RESULTS}

The research aimed to check if some particular observer's features influence the way the image is being observed. The second aim was to check if the content of the image influences gaze patterns. Therefore, we defined three independent features describing the observer: qualification, gender, and age and two independent features describing the image: the level of imperfection and imgGender gender of a person on the image (see Table 1). It was first checked if there are statistically significant differences in these groups for dependent variables (all gaze-related features). According to the Kolmogorov-Smirnov test, all variables were normally distributed. Therefore, for variables with only two possible values (qualification, gender and imgGender) the t-Student test was used, and for variables with more than two possible values (age and level) the ANOVA test was used with Bonferroni correction. For the sake of readability, values of the homogeneity and uniformity were multiplied by $10 e 3$ and $10 e 5$, respectively. 
Table 1. The features taken into account.

\begin{tabular}{ll} 
Feature & Description \\
\hline gender & independent features \\
age & gender of the observer \\
qual & age of the observer \\
level & qualification of the observer (layman, specialist) \\
imgGender & level of imperfection (1-3) \\
\hline & gender of a person on the image \\
\hline fixNum & number of fixations \\
fixAvgDur & average fixation duration \\
sacAvgLen & average saccade length \\
sacLen & summary of saccades length \\
mouthTimeto time before focusing the mouth AOI \\
mouthTime & the overall time of gazing at mouth AOI \\
incisTimeTo & time before focusing the incisors AOI \\
incisTime & the overall time of gazing at incisors AOI \\
rec & reccurence \\
det & determination \\
lam & laminarity \\
corm & center of recurrence mass \\
coverage5 & coverage of the image in 5x5 grid \\
coverage7 & coverage of the image in 7x7 grid \\
coverage10 & coverage of the image in 10x10 grid \\
contrast & GSSP contrast \\
homog & GSSP homogeneity \\
uniformity & GSSP uniformity \\
\hline &
\end{tabular}

\subsection{Observer related variables}

The results analysis starts with discussing features related to the observer, namely: qualification, gender, and age.

Qualification We verified the hypothesis that eye movement patterns are different for specialists and laypeople. Average values of features and standard deviations were calculated, and then the t-Student test was used to decide if the differences are significant. As it is visible in Table 2, this hypothesis has been confirmed as nearly all analyzed features (apart from fixAvgDur) occurred to be significantly different between groups.

It is not surprising that for the chosen AOIs (mouth and teeth), the observation is longer, and timeto values are shorter for specialists. However, it is interesting to notice that other features exhibit significant differences as well. The only exception is uniformity, which is similar for both groups. 
P. Kasprowski et al.

Table 2. Features values - differences for laypeople and specialists.

\begin{tabular}{lccccc}
\hline feature & \multicolumn{7}{c}{ laymen specialists } & $\mathbf{t}(\mathbf{1 8 8 3})$ & $\mathbf{p}$-value \\
\hline fixNum & 14,6 & 15,4 & $-3,289$ & 0,001 & $* *$ \\
fixAvgDur & 345,9 & 359,4 & $-0,704$ & 0,482 & \\
sacAvgLen & 6,9 & 4,7 & 20,024 & 0 & $* * *$ \\
sacLen & 100,2 & 70,6 & 14,976 & 0 & $* * *$ \\
mouthTimeto & 2253,2 & 1054 & 14,12 & 0 & $* * *$ \\
mouthTime & 986 & 1977,4 & $-14,818$ & 0 & $* * *$ \\
incisTimeto & 3991,3 & 2574,8 & 13,573 & 0 & $* * *$ \\
incistime & 428,5 & 962,7 & $-10,353$ & 0 & $* * *$ \\
rec & 13,1 & 21,3 & $-12,178$ & 0 & $* * *$ \\
det & 30,8 & 46,1 & $-9,167$ & 0 & $* * *$ \\
lam & 51,6 & 80,4 & $-14,702$ & 0 & $* * *$ \\
corm & 4,8 & 8,1 & $-8,822$ & 0 & $* * *$ \\
coverage 5 & 0,21 & 0,17 & 13,204 & 0 & $* * *$ \\
coverage7 & 0,14 & 0,11 & 11,043 & 0 & $* * *$ \\
coverage10 & 0,08 & 0,07 & 10,17 & 0 & $* * *$ \\
contrast & 102,4 & 186,6 & 7,23 & 0 & $* * *$ \\
homog & 332,3 & 290,6 & 13,25 & 0 & $* * *$ \\
uniformity & 202,4 & 220,3 & $-2,18$ & 0,03 & $*$ \\
\hline & & & & &
\end{tabular}

Gender The next research question was if the eye movement patterns depend on the gender of the observer. As was already stated in the Introduction, it has been confirmed previously for other datasets. As there were significant differences between specialists and laypeople, we decided to perform the t-Student test independently for both groups. The results are presented in Table 3 and Table 4. There are significant differences in most features depend on the observer's gender in both groups.

Interestingly this time, contrary to the previous case, the values of recurrence and contrast are not significantly different, while the uniformity may be considered as a feature that can reliably distinguish both genders.

Age of the observer Observers were divided into four groups differing with age: (20-30,30-40,40-50,50+). It occurred that for most features the result of ANOVA test revealed significant differences: $F(3,1883)>4.8, p<0.01$. Only for corm feature the effect was less significant with $F(3,1883)=3.268, p=0.021$.

\subsection{Image properties}

The second group of properties included properties related to the observed image - the different level of imperfection (level) and the gender of a person on the image (imgGender). However, this time neither the ANOVA test conducted for level nor t-Student test performed for imgGender showed significant differences in the results (Table 5). 
Table 3. Gender differences for non-specialists.

\begin{tabular}{lccccc}
\hline feature & female & male & $\mathbf{t}(\mathbf{9 5 4})$ & $\mathbf{p}$-value \\
\hline fixNum & 12,9 & 15,6 & $-10,36$ & 0 & $* * *$ \\
fixAvgLen & 422,7 & 297,5 & 9,85 & 0 & $* * *$ \\
sacAvgLen & 7,4 & 6,6 & 4,32 & 0 & $* * *$ \\
sacLen & 95,5 & 103,2 & $-2,62$ & 0,01 & $* *$ \\
mouthTimeTo & 2051,8 & 2380,2 & $-2,35$ & 0,02 & $*$ \\
mouthTime & 1409,1 & 719,2 & 8,31 & 0 & $* * *$ \\
incisTimeTo & 4076 & 3937,9 & 0,93 & 0,35 & \\
incisTime & 587,1 & 328,4 & 4,35 & 0 & $* * *$ \\
rec & 13,4 & 13 & 0,57 & 0,57 & \\
det & 27,9 & 32,7 & $-2,17$ & 0,03 & $*$ \\
lam & 39 & 59,5 & $-7,73$ & 0 & $* * *$ \\
corm & 5,3 & 4,5 & 2,73 & 0,01 & $* *$ \\
coverage5 & 0,2 & 0,22 & $-4,02$ & 0 & $* * *$ \\
coverage7 & 0,13 & 0,14 & $-4,52$ & 0 & $* * *$ \\
coverage10 & 0,08 & 0,08 & $-4,1$ & 0 & $* * *$ \\
contrast & 90,1 & 110,2 & $-1,76$ & 0,08 & \\
homog & 372,6 & 306,8 & 19,62 & 0 & $* * *$ \\
uniformity & 236,12 & 181,1 & 5,89 & 0 & $* * *$ \\
\hline
\end{tabular}

\subsection{Classification}

Disclosing the importance of differences in eye movement features for specialists and non-specialists, males and females, and the observer's age was the motivation factor to verify the possibility of identifying the observer's qualifications based on his/her visual pattern during a single observation of a face.

For this purpose, we applied a simple k-nearest neighbor algorithm with $k=$ 7. The algorithm predicts the given observation's class based on the classification of $k$ nearest training observations. The model was tested using classic 10-fold cross-validation repeated ten times. The results presented in Table 6 confirm the previous findings that the observer's qualification and gender may be estimated using the features defined in Table 1.

The last test was an attempt to predict the observer's age. The task was more difficult than the previous ones because it was 1 out of 4 classification. The confusion matrix for the dataset is presented in Table 7. It may be noticed that the best results were obtained for the participants belonging to the group 40-50. However, the overall accuracy was about $58 \%$.

\section{SUMMARY}

The research presented in this paper aimed to analyze if and how eye movement features depend on the properties of a person being examined and the properties of the image being observed. The detailed analysis confirmed that significant 
P. Kasprowski et al.

Table 4. Gender differences for specialists.

\begin{tabular}{lccccc}
\hline feature & female & male & $\mathbf{t}(\mathbf{9 5 4})$ & $\mathbf{p}$-value \\
\hline fixNum & 13 & 16,4 & $-7,69$ & 0 & $* * *$ \\
fixavgDur & 662,8 & 225,1 & 8,67 & 0 & $* * *$ \\
sacAvgLen & 5,2 & 4,5 & 4,39 & 0 & $* * *$ \\
sacLen & 72,1 & 70 & 0,65 & 0,52 & \\
mouthTimeTo & 893,3 & 1125,2 & $-2,1$ & 0,04 & $*$ \\
mouthTime & 2997 & 1526,1 & 11,58 & 0 & $* * *$ \\
incisTimeTo & 2359,8 & 2669,9 & $-1,87$ & 0,06 & \\
incisTime & 1452,6 & 745,8 & 6,56 & 0 & $* * *$ \\
rec & 21,9 & 20,9 & 0,76 & 0,45 & \\
det & 34,7 & 51,1 & $-6,47$ & 0 & $* * *$ \\
lam & 66,6 & 86,5 & $-6,66$ & 0 & $* * *$ \\
corm & 10,3 & 7,2 & 3,27 & 0 & $* *$ \\
coverage5 & 0,16 & 0,17 & $-1,77$ & 0,08 & \\
coverage7 & 0,11 & 0,11 & $-2,49$ & 0,01 & $*$ \\
coverage10 & 0,06 & 0,07 & $-3,45$ & 0 & $* * *$ \\
contrast & 167,6 & 194,9 & $-1,25$ & 0,21 & \\
homog & 334,2 & 271,3 & 13,36 & 0 & $* * *$ \\
uniformity & 295,8 & 186,8 & 7,07 & 0 & $* * *$ \\
\hline
\end{tabular}

differences in eye movement patterns might be found for qualification and gender. Probably the most interesting finding was that contrast and homogeneity proved to be useful in differentiating the level of expertise, while uniformity depended on the gender of a person.

It was impossible to find any significant dependencies regarding the type of an image - probably because the images represented the same kind of stimulus (face) and were characterized by similar features.

The main limitation of the presented results is the vague meaning of the novel features being analyzed. The semantics of the presented REC and GSSP features is for now not clear. More studies of these features are necessary to define what they represent precisely. The research presented in this paper shows the meaningfulness of these features; however, their values are still not as easy to interpret as average fixation duration or AOI gazing time.

Future work will include tests using other datasets and more in-depth analysis of the novel features. It is planned to check if these features could be used to distinguish a type of observed images or to identify people.

\section{Acknowledgement}

This work was supported by Silesian University of Technology, Rector's ProQuality Grant No: 02/100/RGJ20/0002 and Statutory Research funds of Department of Applied Informatics, Grant No: 02/100/BK_20/0003. 
Table 5. Features values for images containing women and men (imgGender) - the results for specialists.

\begin{tabular}{|c|c|c|c|c|c|}
\hline feature & female & male & $\mathrm{t}(929)$ & p-value & \\
\hline fixNum & 15,4 & 15,3 & 0,39 & 0,7 & \\
\hline fixavgDur & 357,9 & 361,9 & $-0,11$ & 0,91 & \\
\hline sacAvgLen & 4,8 & 4,5 & 1,87 & 0,06 & \\
\hline sacLen & 72,8 & 66,8 & 2,21 & 0,03 & * \\
\hline mouthTimeTo & 1098,3 & 977,4 & 1,14 & 0,26 & \\
\hline mounthTme & 1910,2 & 2093,8 & $-1,58$ & 0,11 & \\
\hline incisTimeTo & 2655,8 & 2434,4 & 1,41 & 0,16 & \\
\hline incisTime & 918,6 & 1039 & $-1,28$ & 0,2 & \\
\hline rec & 20,9 & 21,8 & $-0,74$ & 0,46 & \\
\hline det & 46,3 & 45,8 & 0,18 & 0,86 & \\
\hline lam & 81,4 & 78,8 & 0,86 & 0,39 & \\
\hline corm & 8 & 8,4 & $-0,63$ & 0,53 & \\
\hline coverage 5 & 0,17 & 0,16 & 1,81 & 0,07 & \\
\hline coverage7 & 0,11 & 0,11 & $-0,19$ & 0,85 & \\
\hline coverage10 & 0,07 & 0,07 & 0,84 & 0,4 & \\
\hline contrast & 177,8 & 201,8 & $-1,08$ & 0,28 & \\
\hline homog & 289,1 & 293,2 & $-0,84$ & 0,4 & \\
\hline uniformity & 219,2 & 221,4 & $-0,17$ & 0,86 & \\
\hline
\end{tabular}

\section{References}

1. Anderson, N.C., Bischof, W.F., Laidlaw, K.E., Risko, E.F., Kingstone, A.: Recurrence quantification analysis of eye movements. Behavior research methods 45(3), 842-856 (2013)

2. Blais, C., Jack, R.E., Scheepers, C., Fiset, D., Caldara, R.: Culture shapes how we look at faces. PloS one 3(8), e3022 (2008)

3. Cantoni, V., Galdi, C., Nappi, M., Porta, M., Riccio, D.: Gant: Gaze analysis technique for human identification. Pattern Recognition 48(4), 1027-1038 (2015)

4. Dalton, K.M., Nacewicz, B.M., Johnstone, T., Schaefer, H.S., Gernsbacher, M.A., Goldsmith, H., Alexander, A.L., Davidson, R.J.: Gaze fixation and the neural circuitry of face processing in autism. Nature neuroscience 8(4), 519-526 (2005)

5. Hall, J.K., Hutton, S.B., Morgan, M.J.: Sex differences in scanning faces: Does attention to the eyes explain female superiority in facial expression recognition? Cognition \& Emotion 24(4), 629-637 (2010)

6. Kasprowski, P., Harezlak, K.: Gaze self-similarity plot-a new. Journal of Eye Movement Research 10(3), 1-14 (2017)

7. Kasprowski, P.: Mining of eye movement data to discover people intentions. In: International Conference: Beyond Databases, Architectures and Structures. pp. 355-363. Springer (2014)

8. Kasprowski, P., Harezlak, K.: The second Eye Movements Verification and Identification Competition. In: 2014 IEEE International Joint Conference on Biometrics (IJCB). pp. 1-6. IEEE (2014)

9. Kasprowski, P., Harezlak, K., Fudalej, P., Fudalej, P.: Examining the impact of dental imperfections on scan-path patterns. In: International Conference on Intelligent Decision Technologies. pp. 278-286. Springer (2017) 
Table 6. Results of classification for all two-class features.

\begin{tabular}{lcccc}
\hline feature & accuracy & precision & recall & F1-score \\
\hline qual & $79,4 \%$ & $80,8 \%$ & $78,1 \%$ & $79,4 \%$ \\
qual/fem & $86,4 \%$ & $85,9 \%$ & $83,4 \%$ & $84,7 \%$ \\
qual/male & $78,3 \%$ & $79,8 \%$ & $78,9 \%$ & $79,4 \%$ \\
gender & $77,6 \%$ & $86,8 \%$ & $80,4 \%$ & $83,5 \%$ \\
gender/lay & $80,6 \%$ & $84,6 \%$ & $83,9 \%$ & $84,2 \%$ \\
gender/spec & $78,4 \%$ & $90,6 \%$ & $80,7 \%$ & $85,4 \%$ \\
imGen & $57,0 \%$ & $21,2 \%$ & $35,9 \%$ & $26,7 \%$ \\
imGen/spec & $59,2 \%$ & $27,1 \%$ & $41,2 \%$ & $32,7 \%$ \\
imGen/lay & $57,5 \%$ & $22,2 \%$ & $37,9 \%$ & $28,0 \%$ \\
\hline
\end{tabular}

Table 7. Confusion matrix for age classification for non-specialists.

\begin{tabular}{c|cccc}
\hline & $20-30$ & $30-40$ & $40-50$ & $50+$ \\
\hline $20-30$ & 108 & 50 & 21 & 9 \\
$30-40$ & 88 & 211 & 34 & 27 \\
$40-50$ & 19 & 18 & 220 & 4 \\
$50+$ & 2 & 8 & 2 & 35 \\
\hline
\end{tabular}

10. Kasprowski, P., Harezlak, K., Kasprowska, S.: Development of diagnostic performance \& visual processing in different types of radiological expertise. In: Proceedings of the 2018 ACM Symposium on Eye Tracking Research \& Applications. p. 40. ACM (2018)

11. Liu, S., Quinn, P.C., Wheeler, A., Xiao, N., Ge, L., Lee, K.: Similarity and difference in the processing of same-and other-race faces as revealed by eye tracking in 4-to 9-month-olds. Journal of Experimental Child Psychology 108(1), 180-189 (2011)

12. Mendez, M.F., Mendez, M., Martin, R., Smyth, K.A., Whitehouse, P.J.: Complex visual disturbances in Alzheimer's disease. Neurology 40(3 Part 1), 439-439 (1990)

13. Reingold, E.M., Charness, N., Pomplun, M., Stampe, D.M.: Visual span in expert chess players: Evidence from eye movements. Psychological Science 12(1), 48-55 (2001)

14. Suwa, K., Furukawa, A., Matsumoto, T., Yosue, T.: Analyzing the eye movement of dentists during their reading of ct images. Odontology 89(1), 0054-0061 (2001) 